\title{
miR-376b-5p regulates angiogenesis in cerebral ischemia
}

\author{
LING-JUAN LI, QING HUANG, NING ZHANG, GUI-BIN WANG and YUN-HAI LIU \\ Department of Neurology, Xiangya Hospital of Central South University, Changsha, Hunan 410008, P.R. China
}

Received September 9, 2013; Accepted March 17, 2014

DOI: $10.3892 / \mathrm{mmr} .2014 .2172$

\begin{abstract}
The profile of microRNAs (miRNAs) altered following middle cerebral artery occlusion (MCAO) and miRNAs are involved in angiogenesis following cerebral ischemia. miR-376b-5p was decreased following MCAO, however, whether miR-376b-5p is important in angiogenesis remains to be elucidated. The present study was designed to identify whether miR-376b-5p is involved in angiogenesis following cerebral ischemia and to elucidate the underlying mechanisms. A rat MCAO model was established and quantitative polymerase chain reaction was performed to analyze the mRNA expression level of miR-376b-5p for 1 to 7 days. In addition, the density of microvessels and the relative mRNA and protein levels of hypoxia-inducible factor-1 $\alpha$ (HIF-1 $\alpha$ ), vascular endothelial growth factor A (VEGFA) and Notch1 were measured. The miR-376b-5p mimic or the miR-376b-5p inhibitor were transfected into hypoxic human umbilical vein endothelial cells (HUVECs), and the proliferation, migration and tube formation were measured. To further examine the underlying mechanisms, shRNA was transfected into cells to knock down HIF-1 $\alpha$, and angiogenesis and the expression of associated molecules, including HIF-1 $\alpha$, VEGFA and Notch1 were compared between each group. Our results demonstrated that miR-376b-5p repressed angiogenesis in vivo and in vitro, and miR-376b-5p inhibited angiogenesis in HUVECs by targeting the HIF-1 $\alpha$-mediated VEGFA/Notch1 signaling pathway. These findings provide new insights into angiogenesis therapy for cerebral ischemia.
\end{abstract}

\section{Introduction}

Stroke is a life-threatening disease with a high incidence, high disability and high mortality rate, causing heavy economic burden to society and families. Cerebral infarction accounts for $80 \%$ of the total number of strokes (1). In the pathophysiological process of cerebral infarction, the recovery of the peripheral blood supply is an important factor for nerve cell

Correspondence to: Professor Yun-Hai Liu, Department of Neurology, Xiangya Hospital of Central South University, 87 Xiangya Road, Changsha, Hunan 410008, P.R. China

E-mail: yunhailiu2013168@163.com

Key words: miR-376b-5p, angiogenesis, cerebral ischemia, hypoxia prognosis (2). The clinical treatments for cerebral infarction, include thrombolysis, anticoagulation, blood pressure regulation and microcirculation improvement. The improvement and increase of blood supply in ischemic brain tissue, particularly in the ischemic penumbra, thereby improving energy metabolism of ischemic brain cells and saving neurons is one of the fundamental purposes and fundamental approaches for the treatment of cerebral ischemia.

In recent years, a series of studies have revealed that good collateral circulation is able to reduce the infarct volume and reduce the risk of cerebral infarction recurrence $(3,4)$. Clinical studies have provided preliminary evidence that promoting collateral circulation is able to lead to an improved clinical prognosis (5). Cerebral collateral circulation is blood flow which arrives in the ischemic area through other vessels (collateral or the newly formed vascular anastomosis) when arteries in the brain suffer severe stenosis or occlusion. Therefore, angiogenesis, as an effective method to improve the blood supply to the brain, is key for improving the prognosis of patients with stroke, and has become a major focus of study in recent years. Therapeutic angiogenesis provides a new therapeutic strategy for revascularization in ischemic stroke (6).

A previous study has demonstrated that a variety of microRNAs (miRNAs) are involved in the pathophysiological process of angiogenesis (7). Since the number of miRNAs is large and the understanding of their roles is unclear, the role of miRNAs in the regulation of angiogenesis requires further study and in depth discussion.

Therefore, the present study aimed to examine the role of miRNA-376b-5p in angiogenesis and the mechanisms mediated by the hypoxia-inducible factor-1 $\alpha$ (HIF-1 $\alpha)$-vascular endothelial growth factor (VEGF)-Notch1 pathway in cerebral ischemia. To the best of our knowledge, the present study provides the first demonstration that miR-376b-5p is involved in cerebral ischemia injury and angiogenesis, and also elucidates the regulatory pathway involved.

\section{Materials and methods}

Permanent middle cerebral artery occlusion ( $p M C A O)$ model establishment. This study was approved by the Ethics Committee of Xiangya Medical College, Central South University (Changsha, China). All procedures involving animals were performed in accordance with the NIH Guide for the Care and Use of Laboratory Animals (NIH Publication no. 86-23, revised 1986). A pMCAO model was performed according to a study by Longa et al, however, with 
certain modifications (8). The Sprague-Dawley rats were provided by the Experimental Animal Center of Central South University (Changsha, China). Briefly, 48 male adult Sprague-Dawley rats aged 6-8 weeks old and weighing 250-320 g were anesthetized by intraperitoneal injection of $10 \%$ chloral hydrate $(4 \mathrm{ml} / \mathrm{kg} ;$ Xiangya Hospital of Central South University). Then, the animals were placed in the supine position and, through a $2-\mathrm{cm}$ midline incision in the neck, the right common carotid artery was exposed. Under an operating microscope, the right external carotid artery was isolated and coagulated distal to the bifurcation. A 4-0 nylon suture with a round tip was inserted into the internal carotid artery through the external carotid artery stump until mild resistance was felt to occlude the origin of the middle cerebral artery. Then the wound was closed. Sham surgery animals were anaesthetized and were subjected to artery isolation without MCAO. Neurological function was evaluated following surgery by Longa's score (8). Animals with scores of 1-3 were included. Following the duration of ischemia for 1, 3 and 7 days, rats in the MCAO group were anesthetized and perfused transcardially with sodium chloride followed by $4 \%$ paraformaldehyde. Decapitation was performed to remove the brains quickly.

TTC staining. Male adult Sprague-Dawley rats were anesthetized by intraperitoneal injection of $10 \%$ chloral hydrate ( $4 \mathrm{ml} / \mathrm{kg}$ ) and decapitation was performed to remove the brains quickly. The brains were refrigerated at $-80^{\circ} \mathrm{C}$ for $5 \mathrm{~min}$ and then cut into sections of $\sim 2 \mathrm{~mm}$ thickness. The brain slices were added to $2 \%$ TTC (Sigma, St. Louis, $\mathrm{MO}$, USA) at $37^{\circ} \mathrm{C}$ for $20 \mathrm{~min}$ and then stirred to stain completely. Following staining, the brain slices were fixed in $4 \%$ paraformaldehyde solution for $24 \mathrm{~h}$.

Immunohistochemistry. The brains were fixed in 4\% paraformaldehyde for $24 \mathrm{~h}$ prior to being embedded in paraffin. For immunohistochemistry, serial sections $(4 \mu \mathrm{m})$ were mounted on slides to dewax and rehydrate. Following antigen retrieval under high pressure in citrate buffer, goat serum (ZSGB-BIO Company, Beijing, China) was used to inhibit nonspecific staining and $3 \%$ hydrogen peroxide was added to inhibit endogenous peroxidase activity. The slides were incubated with primary antibodies [chicken polyclonal to von Willebrand factor (vWF) antibody; Abcam, Cambridge, MA, USA, 1:200] at $37^{\circ} \mathrm{C}$ for $1 \mathrm{~h}$ followed by incubation with a secondary antibody [goat anti-chicken IgY (HRP), Abcam]. The horseradish peroxidase reaction was detected using the DAB staining kit (Maixin, Fuzhou, Fujian, China) according to the manufacturer's instructions. The slides were counterstained with hematoxylin and eosin. All the slides were visualized under a microscope (E200; Nikon, Tokyo, Japan).

Cell culture. Human umbilical vein endothelial cells (HUVECs) were purchased from American Type Culture Collection (Manassas, VA, USA). The cells in the normal group were maintained in DMEM (Gibco-BRL, Grand Island, NY, USA), supplemented with $10 \%$ newborn calf serum (Invitrogen Life Technologies, Carlsbad, CA, USA) at $37^{\circ} \mathrm{C}$ in a humidified $5 \% \mathrm{CO}_{2}, 95 \% \mathrm{O}_{2}$ atmosphere. The medium was adjusted every 2-3 days and the cells were routinely split into two cultures after 4 days. The cells in the hypoxia group were maintained in DMEM without serum at $37^{\circ} \mathrm{C}$ in a humidified $5 \% \mathrm{CO}_{2}, 94 \% \mathrm{~N}_{2}, 1 \% \mathrm{O}_{2}$ atmosphere.

Transfection. Prior to transfection of the miR-376b-5p mimic/inhibitor and HIF-1 $\alpha$ shRNA, a pre-experiment was performed to select the most effective HIF-1 $\alpha$ shRNA fragment. HUVECs were transfected using Lipofectamine 2000 (Invitrogen Life Technologies) according to the manufacturer's instructions. After $6 \mathrm{~h}$, the cells were washed and maintained in the cultures for at least $24 \mathrm{~h}$ for further analysis.

MTT assay. HUVECs were seeded into 96-well plates and allowed to grow for the appropriate times, and then $10 \mu \mathrm{l}$ of MTT solution (0.5 mg/ml; Beyotime, Shanghai, China) was added to each well and incubated at $37^{\circ} \mathrm{C}$ for $4 \mathrm{~h}$. DMSO $(150 \mu \mathrm{l}$; Sigma) was added and incubated for $15 \mathrm{~min}$ to dissolve the formazan crystals. The absorbance was measured at $570 \mathrm{~nm}$ by a microplate reader (ELx800NB; BioTek Instruments, Inc., Winooski, VT, USA).

Tube formation assay. The tube formation assay was performed using HUVECs on Matrigel. BD Matrigel matrix (200 $\mu \mathrm{l}$; BD Biosciences, Franklin Lakes, NJ, USA) was added to 24-well plates on ice. Then, it was incubated at $37^{\circ} \mathrm{C}$ in $5 \% \mathrm{CO}_{2}$ for $30 \mathrm{~min}$ to allow gel formation. HUVECs were digested and the cells were suspended at a density of $4 \times 10^{5} / \mathrm{ml}$. Following gel solidification for $30 \mathrm{~min}, 50 \mu \mathrm{l}$ of cell suspension was added to each well and the cell culture medium was compensated to $1 \mathrm{ml}$. The plates were incubated at $37^{\circ} \mathrm{C}$ in $5 \% \mathrm{CO}_{2}$. Following incubation for 2, 4 and $8 \mathrm{~h}$, tube formation was observed using an inverted microscope (TS100; Nikon). Image-Pro Plus 5.0 (BD Biosciences) software was used and 10 different visual fields were selected to analyze the tube lengths.

Transwell migration assay. The cell migration experiments were performed using a 6 -well Transwell system $(8 \mu \mathrm{m}$; Corning, Inc., Corning, NY, USA). The cells were digested, following washing with phosphate-buffered saline, and counted and resuspended in media without serum to obtain a cell density of $5 \times 10^{4} / \mathrm{ml}$. Cell media with fetal bovine serum ( $1 \mathrm{ml}$; Invitrogen Life Technologies) was added to the lower chamber and $2 \mathrm{ml}$ of cell suspension was added to the Transwell plates. Following incubation for 12, 24 and 48 h, the upper chamber was fixed in $95 \%$ ethanol for $15 \mathrm{~min}$ and stained with hematoxylin for $10 \mathrm{~min}$. The cells were counted under the TS100 microscope.

Quantitative polymerase chain reaction ( $q P C R)$. Total RNAs were isolated from cerebral infarction or HUVECs using TRIzol reagent (Invitrogen Life Technologies). According to the manufacturer's instructions, $2 \mu \mathrm{g}$ of total RNA was used for reverse transcription using a cDNA synthesis kit (RevertAid $^{\mathrm{TM}}$ First Strand cDNA Synthesis kit; Fermentas, Vilnius, Lithuania). The samples were quantified by qPCR in a 7300 Sequence Detection system (Applied Biosystems, Foster City, CA, USA) using an SYBR-Green PCR kit (Applied Biosystems). The relative expression level was calculated by the comparative CT method. The sequences of the amplified miRNA transcripts were as follows: rno-miR-376b-5p, 


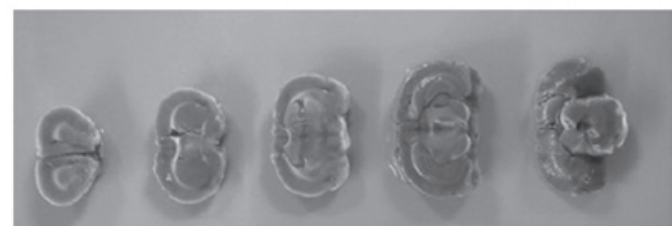

sham

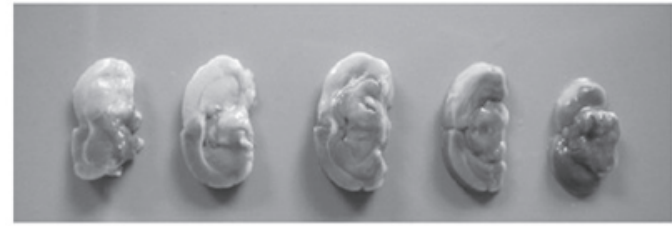

3 days

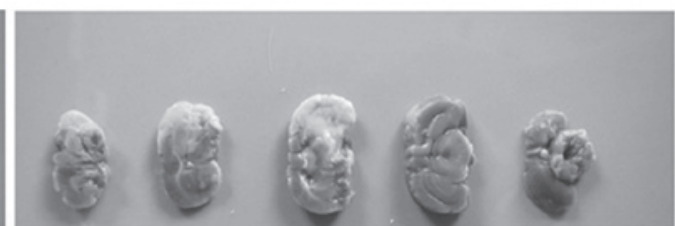

1 day

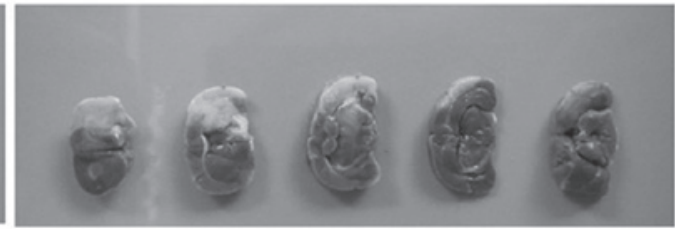

7 days

Figure 1. Representative images of TTC staining of rat brain slices following MCAO. MCAO, middle cerebral artery occlusion.

\section{5'-GUGGAUAUUCCUUCUAUGGUUA-'3; hsa-miR-376b-5p, 5'-CGUGGAUAUUCCUUCUAUGUUU-3'.}

Western blot analysis. The protein samples were loaded onto SDS-PAGE (Beyotime) and separated by electrophoresis. Then, proteins were transferred onto a PVDF membrane (EMD Millipore Corporation, Billerica, MA, USA) and incubated in $1 \%$ bovine serum albumin (Amresco Inc., Solon, $\mathrm{OH}, \mathrm{USA}$ ) at $37^{\circ} \mathrm{C}$ for $1 \mathrm{~h}$. The blots were probed with specific primary antibodies (rabbit polyclonal to Notch1 and rabbit polyclonal to VEGFA, Abcam; rabbit monoclonal to HIF-1 $\alpha$, EMD Millipore Corporation; mouse monoclonal to GAPDH, Santa Cruz Biotechnology, Inc., Santa Cruz, CA, USA) and then the membranes were incubated with horseradish peroxidase-conjugated secondary antibody at $37^{\circ} \mathrm{C}$ for $1 \mathrm{~h}$. The signals were visualized using a chemiluminescence-based detection system (ECL Western Blotting kit; Pierce Biotechnology, Inc., Rockford, IL, USA).

Statistical analysis. Statistical analysis was performed using SPSS 13.0 software (SPPS, Inc., Chicago, IL, USA). Student's t-test was used for analysis. Data are representative of at least three independent experiments and are presented as the means \pm standard deviation. $\mathrm{P}<0.05$ was considered to indicate a statistically significant difference.

\section{Results}

TTC staining certifies that the PMCAO model was established successfully. As shown in Fig. 1, TTC staining demonstrated that in the pMCAO groups, the infarction area was white and the normal brain tissue was red (dark grey in Fig. 1). The infarction scope in the cerebral tissue was consistent with the blood-supply region controlled by the middle cerebral artery. The brain tissue in the sham group was uniformly red. The pMCAO model was established successfully.

vWF expression increases following MCAO. vWF immunohistochemistry images of cerebral infarction following MCAO are shown in Fig. 2. vWF-positive cells were confined to the endothelial cells in microvessels of the infarction area. The results demonstrated that the vWF-positive cell number
A

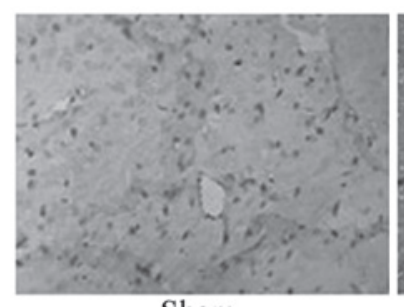

Sham

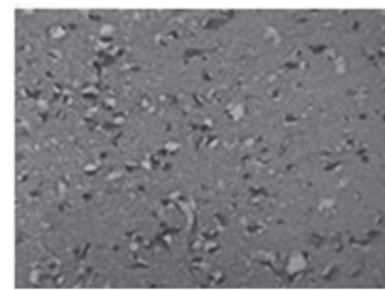

3 days

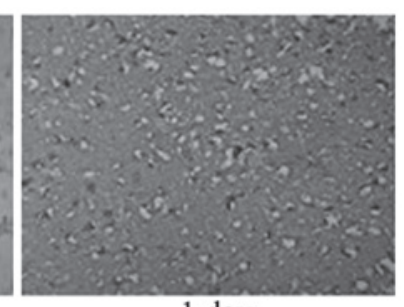

1 day

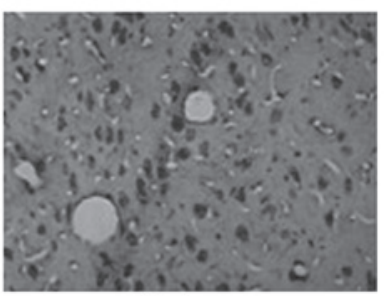

7 days
B

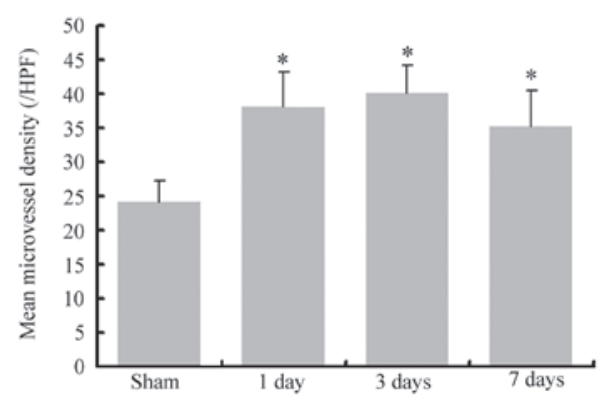

Figure 2. vWF immunohistochemistry of the rat brain following MCAO. (A) Representative images of brains stained with vWF antibody to identify capillaries (magnification, $\mathrm{x} 400$ ). (B) Mean microvessel density at different time points following MCAO. Microvessels were counted from 10 fields of the ischemic area under a microscope (magnification, $\mathrm{x} 400$ ). Data are expressed as the mean $\pm \mathrm{SD}$. ${ }^{*} \mathrm{P}<0.05$, compared with the sham group. vWF, von Willebrand factor; MCAO, middle cerebral artery occlusion; HPF, high-power field.

significantly increased following MCAO compared with the sham group $(\mathrm{P}<0.05)$. These data suggested that angiogenesis was induced following cerebral ischemia.

miR-376b-5p is downregulated following MCAO. To examine the expression changes of miR-376b-5p in cerebral ischemia, qPCR was used to analyze the relative mRNA expression level 
of miRNA-376b-5p following MCAO. The relative mRNA level in the sham group was designated as 1 and it was revealed that miRNA-376b-5p was gradually downregulated 1 day $(0.79 \pm 0.03)$ after MCAO $(\mathrm{P}<0.05)$ and then decreased rapidly to the minimum 7 days $(0.06 \pm 0.01)$ after MCAO $(\mathrm{P}<0.01)$. Therefore, miRNA-376b-5p appears to be involved in cerebral ischemia.

Alterations in the mRNA and protein level of HIF-1 $\alpha$, VEGFA and Notchl following MCAO. To investigate the basis for angiogenesis following cerebral ischemia, the present study examined the relative expression of HIF-1 $\alpha$, VEGFA and Notch1 at the mRNA and protein level following MCAO. As shown in Fig. 3, qPCR and western blotting results indicated that the mRNA and protein expression level in the sham group was low, and it was significantly increased from baseline 1 day after MCAO $(\mathrm{P}<0.05)$ and continued to increase up to 7 days $(\mathrm{P}<0.01)$. In addition, the results demonstrated that the alterations in VEGFA and Notch1 at the mRNA and protein level were significantly increased from 1 day after MCAO $(\mathrm{P}<0.05)$ and remained at high levels until 7 days $(P<0.01)$. These data indicated that HIF-1 $\alpha$, VEGFA and Notch1 are involved in angiogenesis following cerebral ischemia.

Hypoxia downregulates miR-376b-5p in HUVECs. miR-376b-5p was suggested to be downregulated in rat cerebral ischemia. HUVECs were used as an in vitro model to further examine whether hypoxia regulates the expression of miR-376b-5p. HUVECs were grown either in normoxia or hypoxia for different time periods ( 24 or $48 \mathrm{~h}$ ) and alterations in miR-376b-5p levels were determined by qPCR. The results were consistent with the in vivo study; miR-376b-5p levels were decreased in hypoxia for 24 and $48 \mathrm{~h}$ compared with those in normoxia ( $\mathrm{P}<0.05$; Fig. $4 \mathrm{~A})$.

Hypoxia induces angiogenesis in HUVECs. In the in vivo study, angiogenesis was induced following MCAO and the role of hypoxia in angiogenesis in vitro was further investigated. To examine whether hypoxia was able to affect the viability of HUVECs, an MTT assay was performed. Compared with the normoxia group, the viability of HUVECs was repressed in the hypoxia group at $12 \mathrm{~h}$ and then increased up to $48 \mathrm{~h}$ (Fig. 4B). Since tube formation and cell migration were important processes in angiogenesis, the tube length and migrated cell number of HUVECs in hypoxia were further examined. The present study revealed that differences in tube lengths at $2 \mathrm{~h}$ between the normoxia and hypoxia group were not significant $(4.73 \pm 0.88$ vs. $5.69 \pm 1.02 \mu \mathrm{m} ; \mathrm{P}>0.05)$; however, with time, the lengths of the tubes increased in the hypoxia group at 4 and $8 \mathrm{~h}$ compared with the normoxia group ( $4 \mathrm{~h}: 37.31 \pm 7.98$ vs. $22.97 \pm 5.12 \mu \mathrm{m}$; 8 h: $85.43 \pm 19.11$ vs. $53.32 \pm 9.12 \mu \mathrm{m}$; P<0.05) (Fig. 4C). The cell migration ability was investigated by Transwell migration assay and it was revealed that the difference in the migration cell number between normoxia and hypoxia groups was not clear at $12 \mathrm{~h}(22 \pm 5$ vs. $23 \pm 4$; $\mathrm{P}>0.05)$; however, at 24 and $48 \mathrm{~h}$, the migration cell number in the hypoxia group significantly increased compared with the normoxia group ( $24 \mathrm{~h}$ : $62 \pm 9$ vs. $25 \pm 6$; $\mathrm{P}<0.05 ; 48$ h: $71 \pm 15$ vs. $34 \pm 8$; $\mathrm{P}<0.01$ ) (Fig. $4 \mathrm{D}$ ).

miR-376b-5p represses angiogenesis in vitro. To further characterize the involvement of miR-376b-5p in angiogenesis
A

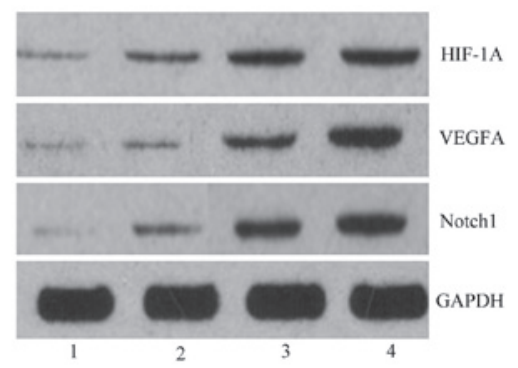

B
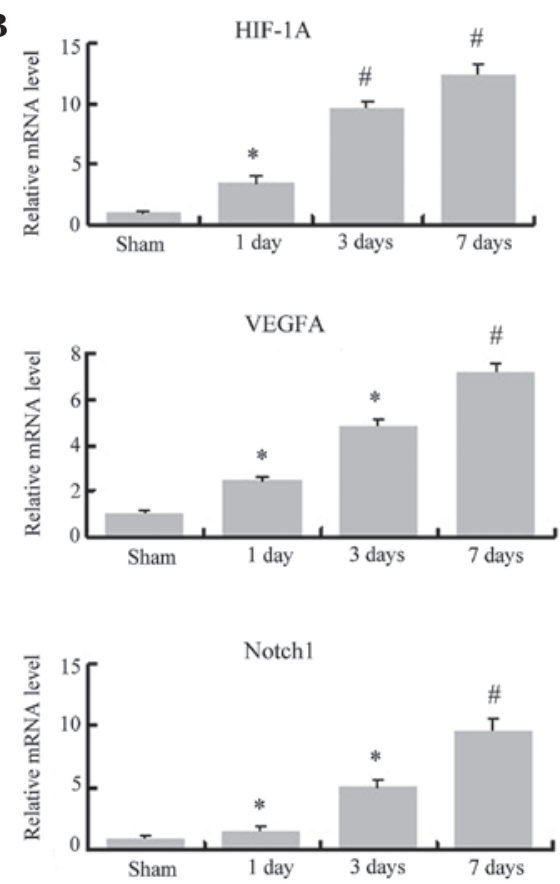

C
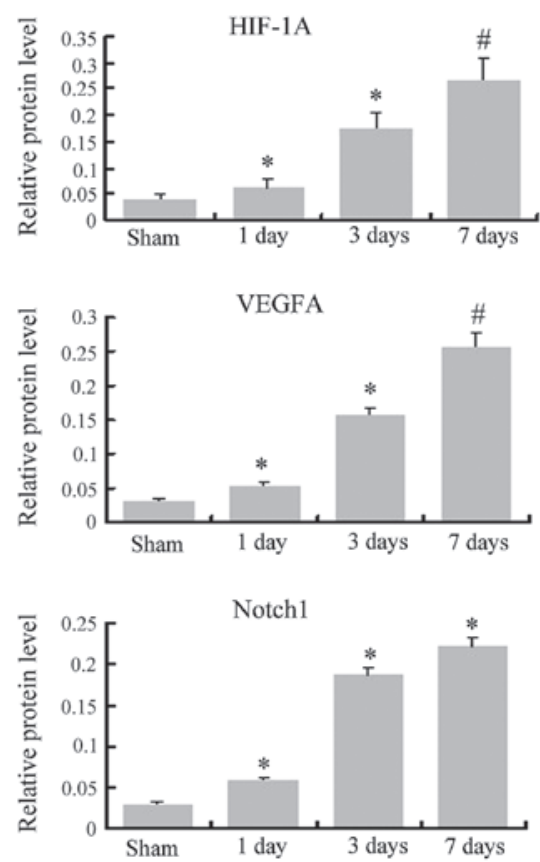

Figure 3. Changes in HIF-1 $\alpha$, VEGFA and Notch1 at the mRNA and protein levels following MCAO. (A) Representative images of western blot analysis for HIF-1 $\alpha$, VEGFA, Notch1 and GAPDH at the indicated time points following MCAO. 1, sham; 2, MCAO 1 day; 3, MCAO 3 days; 4, MCAO 7 days. (B) Relative mRNA levels for HIF-1 $\alpha$, VEGFA and Notch1, normalized to $\beta$-actin. (C) Relative protein expression levels for HIF-1 $\alpha$, VEGFA and Notch1, normalized to GAPDH. " $\mathrm{P}<0.05$, compared with the sham group. ${ }^{\#} \mathrm{P}<0.01$, compared with the sham group. MCAO, middle cerebral artery occlusion; HIF-1 $\alpha$, hypoxia-inducible factor-1 $\alpha$; VEGFA, vascular endothelial growth factor A. 
A

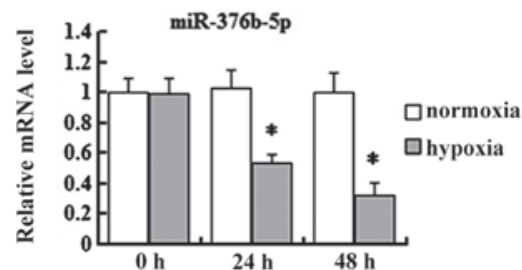

C

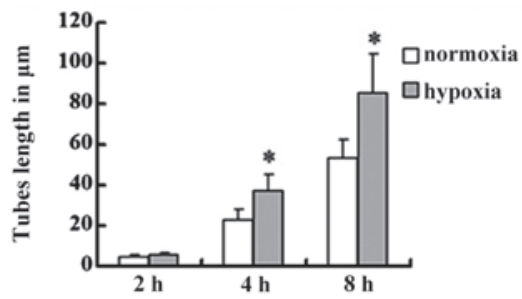

B

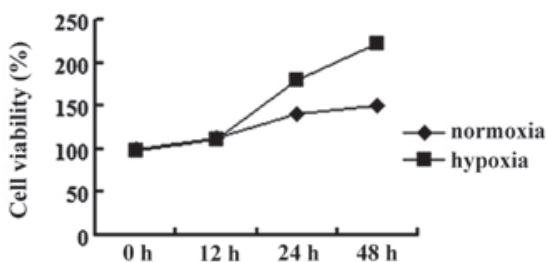

D

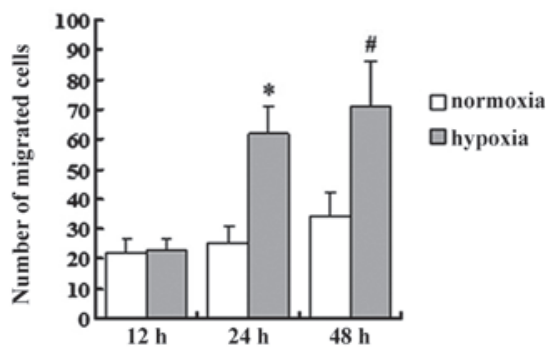

Figure 4. Hypoxia downregulates miR-376b-5p and induces angiogenesis in HUVECs. (A) Relative mRNA level for miR-376b-5p in normoxic and hypoxic HUVECs, normalized to U6. Data are shown as the mean $\pm \mathrm{SD}$. " $\mathrm{P}<0.05$, compared with the normoxia group. (B) MTT assay of HUVECs in normoxia and hypoxia. (C) Tube length of HUVECs in normoxia and hypoxia. Data are shown as the mean $\pm \mathrm{SD}$. ${ }^{*} \mathrm{P}<0.05$, compared with the normoxia group. (D) Migrated cell numbers of HUVECs in normoxia and hypoxia. Migrated cell numbers are shown as the mean \pm SD. "P< $<0.05$, compared with the normoxia group. ${ }^{\#}<<0.01$, compared with the normoxia group. HUVECs, human umbilical vein endothelial cells; miR, microRNA.

A

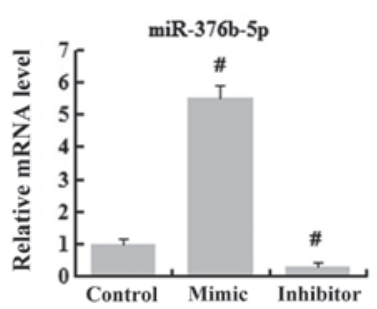

C

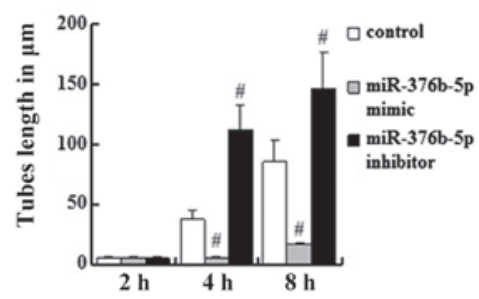

B

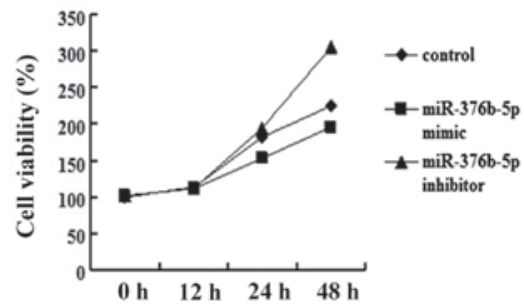

D

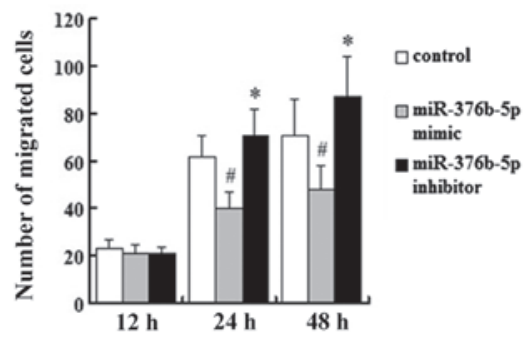

Figure 5. miR-376b-5p represses angiogenesis in vitro. (A) Relative mRNA level for miR-376b-5p in hypoxic HUVECs following transfection with the miR-376b-5p mimic/inhibitor, normalized to U6. Data are shown as the mean $\pm \mathrm{SD} .{ }^{*} \mathrm{P}<0.01$, compared with the control. (B) MTT assay of HUVECs following transfection with the miR-376b-5p mimic/inhibitor. (C) Tube length of HUVECs following transfection with the miR-376b-5p mimic/inhibitor. Data are shown as the mean \pm SD. ${ }^{~} \mathrm{P}<0.01$, compared with the control. (D) Migrated cell numbers of HUVECs following transfection with the miR-376b-5p mimic/inhibitor. Migrated cell numbers are shown as the mean \pm SD. ${ }^{~} \mathrm{P}<0.05$, compared with the control. ${ }^{~} \mathrm{P}<0.01$, compared with the control. HUVECs, human umbilical vein endothelial cells; miR, microRNA.

following cerebral ischemia, the miR-376b-5p mimic and the miR-376b-5p inhibitor were transfected into HUVECs to study whether the expression level of miR-376b-5p was able to affect the process of angiogenesis in response to ischemia injury. The transfection efficiency of the miR-376b-5p mimic group and the miR-376b-5p inhibitor group was $\sim 80 \%$. The relative mRNA level of miR-376b-5p in hypoxic HUVECs transfected with negative control miRNA (miR-NC) was designated to be 1 . The mRNA level of miR-376b-5p increased significantly in miR-376b-5p mimic-transfected HUVECs $(5.54 \pm 0.38)$ compared with miR-NC-transfected cells $(\mathrm{P}<0.01)$, and the level of miR-376b-5p mRNA decreased significantly in the miR-376b-5p inhibitor group $(0.3 \pm 0.13)$ compared with the miR-NC group $(\mathrm{P}<0.01)$ (Fig. 5A). Using HUVECs in hypoxia with miR-NC-transfected cells as the control, the data suggested that the miR-376b-5p mimic was able to significantly repress hypoxia-induced increases in the cell viability of HUVECs; however, the miR-376b-5p inhibitor enhanced hypoxia-induced increases in the cell viability of HUVECs (Fig. 5B). These results indicated that miR-376b-5p represses the cell viability of HUVECs in hypoxia. Next, the present study investigated the tube lengths in HUVECs and revealed that the miR-376b-5p mimic and miR-376b-5p inhibitor did not affect tube formation at $2 \mathrm{~h}(\mathrm{P}>0.05)$. However, with 
time, the tube lengths decreased in the miR-376b-5p mimic group at 4 and $8 \mathrm{~h}$ compared with the miR-NC group $(\mathrm{P}<0.01)$ while the miR-376b-5p inhibitor reversed this decrease $(\mathrm{P}<0.01)$ (Fig. 5C). In addition, the present study examined whether the expression level of miR-376b-5p was able to affect cell migration. The results illustrated that the miR-376b-5p mimic significantly decreased the migration cell number compared with the hypoxia group $(\mathrm{P}<0.01)$ and this effect was reversed by the miR-376b-5p inhibitor $(\mathrm{P}<0.05)$ (Fig. 5D). Overall, these results suggest that miR-376b-5p represses angiogenesis in hypoxic HUVECs.

miR-376b-5p represses angiogenesis via the HIF-1 $\alpha$ target gene. To further examine the role of miR-376b-5p in angiogenesis during hypoxia and its mechanism of regulation, the present study predicted the targets of miR-376b-5p by PicTar (http:// pictar.mdc-berlin.de/) and focused on HIF-1 $\alpha$. The protein expression of HIF-1 $\alpha$ was rare in normoxia, however, it was induced increasingly under hypoxia $(0.035 \pm 0.005$ vs. $0.146 \pm 0.049 ; \mathrm{P}<0.05)$. shRNA against HIF-1 $\alpha$ was transfected into hypoxic HUVECs, as shown in Fig. 6A and, following transfection, HIF-1 $\alpha$ significantly decreased at the protein level $(\mathrm{P}<0.01)$. The MTT assay revealed that cell viability in the shRNA + inhibitor group was lower than that of the inhibitor group and the hypoxia group at 24 and $48 \mathrm{~h}$ (Fig. 6B). The tube formation experiment demonstrated that the length of tubes at $8 \mathrm{~h}$ in the shRNA + inhibitor group was decreased compared with the inhibitor group, also the tube lengths in the shRNA groups were decreased compared with the hypoxia group (Fig. 6C). Furthermore, the migration cell number in the shRNA groups decreased at $24 \mathrm{~h}$ compared with the mimic/inhibitor groups and hypoxia group (Fig. 6D). The same results were also observed in those at $4 \mathrm{~h}$ (data not shown). All these data suggested that miR-376b-5p is able to directly bind to the HIF-1 $\alpha$ gene, which is important in angiogenesis.

$m i R-376 b-5 p$ represses the expression of angiogenesis via the HIF-1 $\alpha$ mediated VEGFA-Notchl pathway. To confirm that miR-376b-5p represses angiogenesis during hypoxia through the HIF-1 $\alpha$-VEGFA-Notch1 pathway, VEGFA and Notch1 relative mRNA and protein levels were also measured by qPCR and western blot analysis. VEGFA relative mRNA and protein level in the hypoxia group were higher than in the normoxia group $(0.049 \pm 0.015$ vs. $0.137 \pm 0.041$ for protein; $\mathrm{P}<0.05)$. Following transfection of the mimic or inhibitor for $48 \mathrm{~h}$, the miR-376b-5p mimic reduced the relative mRNA and protein levels of VEGFA compared with the miR-NC group $(0.070 \pm 0.022$ vs. $0.118 \pm 0.029$ for protein; $\mathrm{P}<0.05)$. The miR-376b-5p inhibitor significantly enhanced hypoxia-induced increases in the relative mRNA and protein levels of VEGFA $(0.246 \pm 0.074$ vs. $0.118 \pm 0.029$ for protein; $\mathrm{P}<0.05)$. The mRNA and protein levels of Notch1 also increased in the hypoxia group compared with the normoxia group $(0.055 \pm 0.015$ vs. $0.140 \pm 0.040$ for protein; $\mathrm{P}<0.05)$ and the miR-376b-5p mimic reduced the relative mRNA and protein levels of Notch1 to low levels $(\mathrm{P}<0.05)$. The miR-376b-5p inhibitor had an opposite effect and the relative mRNA and protein levels of Notch1 were significantly increased in the miR-376b-5p inhibitor group compared with that in the miR-NC group (NC, $0.128 \pm 0.033$; mimic,
A
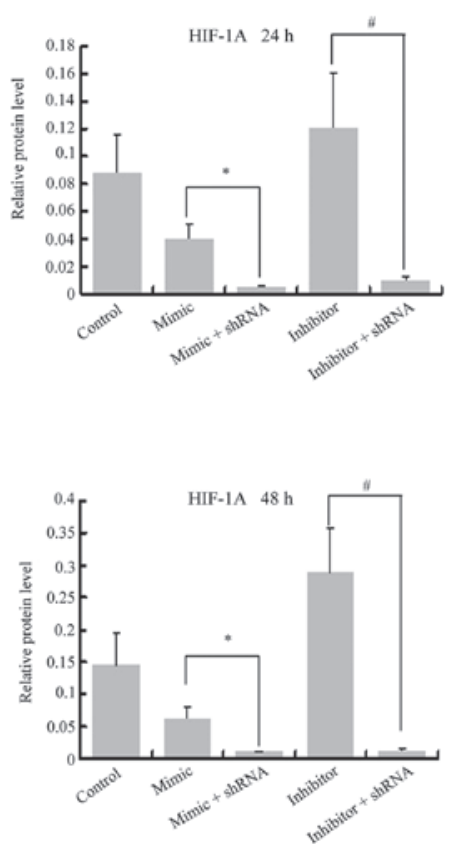

B

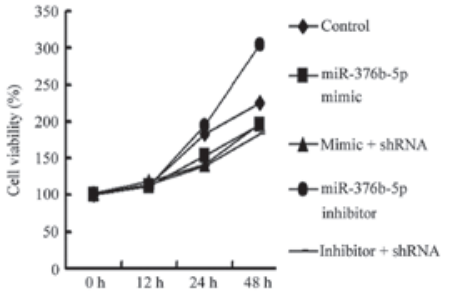

C

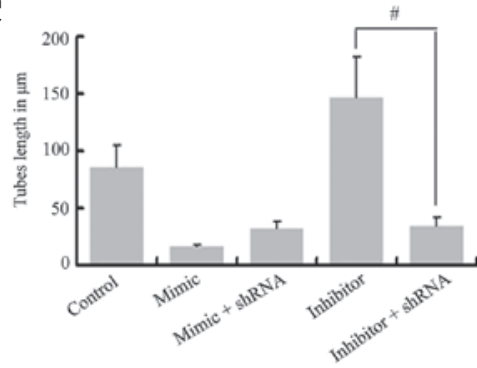

D

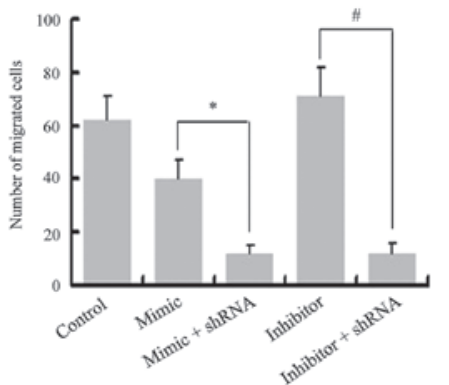

Figure 6. miR-376b-5p represses angiogenesis via the HIF-1 $\alpha$ target gene. (A) HIF-1 $\alpha$ protein expression level following transfection with the miR-376b-5p mimic/inhibitor and HIF-1 $\alpha$ shRNA in hypoxic HUVECs determined by western blot analysis. The hypoxic HUVECs transfected with miR-NC and NC-shRNA were used as the control. Values are shown as the mean \pm SD. ${ }^{*} \mathrm{P}<0.05$ and ${ }^{*} \mathrm{P}<0.01$. (B) MTT assay of HUVECs following transfection with the miR-376b-5p mimic/inhibitor and HIF-1 $\alpha$ shRNA. (C) Tube length of HUVECs $8 \mathrm{~h}$ following transfection with the miR-376b-5p mimic/inhibitor and HIF-1 $\alpha$ shRNA. Data are shown as the mean \pm SD. ${ }^{\#} \mathrm{P}<0.01$. (D) Migrated cell numbers of HUVECs $24 \mathrm{~h}$ following transfection with the miR-376b-5p mimic/inhibitor and HIF-1 $\alpha$ shRNA evaluated by a Transwell migration assay. Migrated cell numbers are shown as the mean \pm SD. ${ }^{*} \mathrm{P}<0.05$ and ${ }^{\#} \mathrm{P}<0.01$. HIF-1 $\alpha$, hypoxia-inducible factor- $1 \alpha$; HUVECs, human umbilical vein endothelial cells; shRNA, small hairpin RNA; NC, negative control; miR, microRNA. 
$0.086 \pm 0.028$; inhibitor, $0.201 \pm 0.062$ for protein; $\mathrm{P}<0.05$ ). The relative mRNA and protein levels of VEGFA in the shRNA groups were lower than those of the mimic/inhibitor groups, and they were also lower than those in the hypoxia group (Fig. 7A). The results also demonstrated that the relative mRNA and protein levels of Notch1 decreased in the shRNA groups compared with the mimic/inhibitor groups and hypoxia group (Fig. 7B). These results suggest that miR-376b-5p downregulates VEGFA and Notch1 expression at the mRNA and protein levels and HIF-1 $\alpha$ mediated this effect.

\section{Discussion}

miRNAs are $\sim 22 \mathrm{nt}$ long non-coding RNAs, which are widely present in eukaryotic organisms. They control mRNA levels and function by binding to the 3'-UTR (9-13). The binding of miRNAs is considered to either degrade the mRNAs or repress translation (14-15). miRNAs are involved in numerous complex physiological processes, including growth and development, organogenesis, cell proliferation and apoptosis. Kulshreshtha et al first reported that hypoxia was able to induce expression changes in miRNAs in cells in 2007 (16). Then, a number of studies revealed that the expression profiles of miRNAs also altered following ischemia and hypoxia in certain organs, including the retina, myocardium and hippocampus (17-19).

In a study by Dharap et al (20), the authors profiled miRNAs following transient MCAO in the adult rat brain. The expression of 238 miRNAs were evaluated between $3 \mathrm{~h}$ and 3 days. Compared with the sham group, eight miRNAs were increased and 12 miRNAs were decreased in at least four out of five reperfusion time points. These studies indicate a critical role of miRNAs in controlling mRNA transcription and translation in the post-ischemic brain. Among the 12 decreased miRNAs, rno-miR-376b-5p was demonstrated to be decreased from $3 \mathrm{~h}$ to 3 days by miRNA microarray analysis. The present study focused on the expression of miR-376b-5p using qPCR.

In our in vivo study, a rat pMCAO model was constructed and the mRNA expression level of miR-376b-5p in the cerebral infarct area was observed from 1 to 7 days using qPCR. The results demonstrated that rno-miR-376b-5p altered following focal ischemia. The mRNA expression level decreased compared with the sham group, which was consistent with miRNA microarray analysis in the study by Dharap et al (20). In our in vitro study, the mRNA expression level of miR-376b-5p in the hypoxia group was downregulated compared with the normoxia group. The results in vivo and in vitro suggested that miR-376b-5p is involved in the regulation of the pathophysiological process following ischemic brain injury.

Angiogenesis is a process involving the proliferating, remodeling and sprouting of endothelial cells and subsequent formation of new blood vessels from pre-existing vasculature (21). Certain insults, including brain trauma and ischemia were able to induce angiogenesis $(22,23)$.

vWF is a specific surface marker of endothelial cells. In the current study, for angiogenesis analysis in vivo, immunocytochemistry was used with the vWF antibody to label microvessels in the cerebral infarct area following MCAO.
A
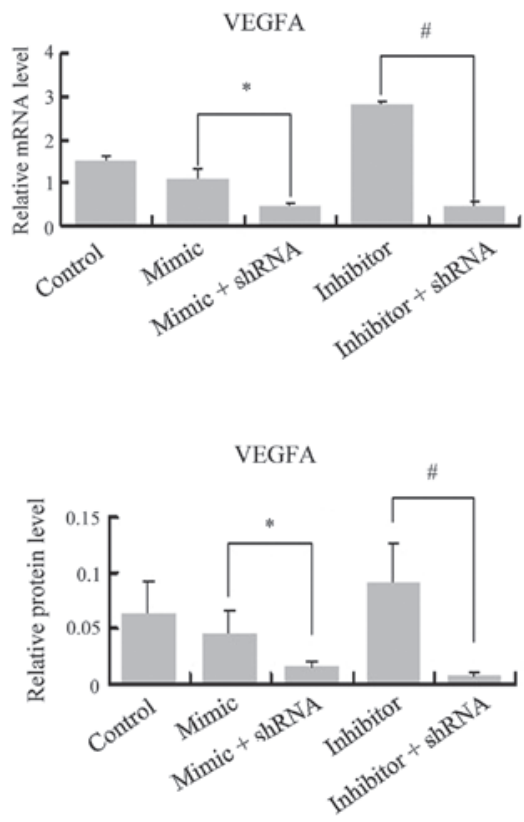

B
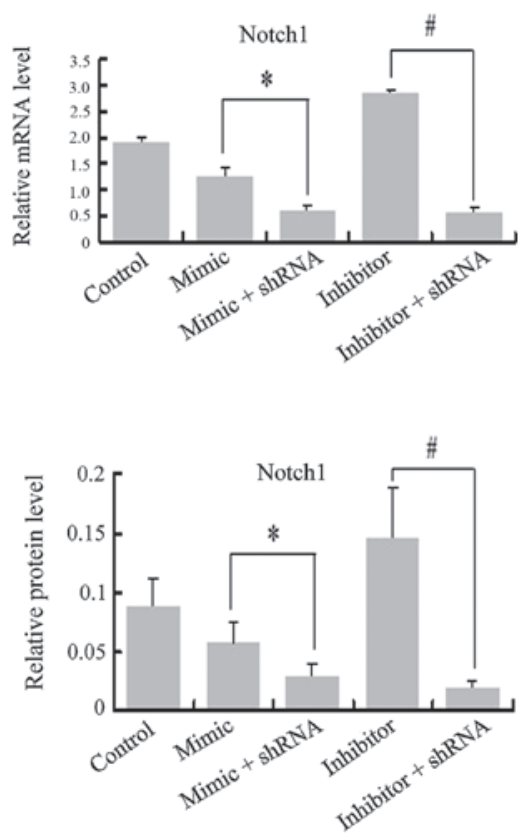

Figure 7. Effect of the miR-376b-5p mimic/inhibitor and HIF-1 $\alpha$ shRNA on the expression of VEGFA and Notch1. (A) VEGFA expression level; (B) Notch1 expression level following transfection with the miR-376b-5p mimic/inhibitor and HIF-1 $\alpha$ shRNA in hypoxic HUVECs determined by quantitative polymerase chain reaction and western blot analysis. The hypoxic HUVECs transfected with miR-NC and NC-shRNA were used as the control. Values are shown as the mean $\pm \mathrm{SD} .{ }^{*} \mathrm{P}<0.05 ;{ }^{~} \mathrm{P}<0.01$. HIF- $1 \alpha$, hypoxia-inducible factor-1 $\alpha$; shRNA, small hairpin RNA; VEGFA, vascular endothelial growth factor A; HUVECs, human umbilical vein endothelial cells; NC, negative control; miR, microRNA.

The in vivo results suggested that the mean microvessel density was increased 1, 3 and 7 days following MCAO, therefore, it confirmed that responsive angiogenesis was induced following cerebral ischemia. It has been demonstrated that the proliferation, migration and morphological differentiation of endothelial cells are important in angiogenesis. In our in vitro study, the results suggested that the proliferation, migration and tube formation of HUVECs were increased under hypoxia. These results also verified that angiogenesis 
was induced following hypoxia, which was consistent with the results in vivo.

Angiogenesis is regulated by activator and inhibitor molecules and signaling pathways (24). These growth factors and kinases affect vascular endothelial cells and smooth muscle cells, stimulating the migration, proliferation and differentiation of endothelial cells, and then new blood vessels form by the sprouting of new capillaries.

VEGFA is the most important activator molecule in the process of angiogenesis. VEGFA is important in angiogenesis following cerebral ischemia (25). It has been revealed that the expression of VEGFA is closely associated with microvessel density and new vascular density in tissue; VEGFA has been demonstrated to participate in the capillary formation and improve the blood flood surrounding the infarction area (26). VEGFA also demonstrated a neuroprotective effect by improving local microcirculation, promoting the injured nerve generation and neural proliferation (27-31).

Numerous studies have confirmed that the Notch signaling system is important in the proliferation and differentiation of endothelial cells. There are four Notch genes in mammals, encoding four Notch receptors, Notch 1, Notch 2, Notch 3 and Notch 4, and five Notch ligands, DII1, DII3, DII4, Jagged 1 and Jagged 2. The Notch signaling system is involved in angiogenesis $(32,33)$. Previous studies have demonstrated that the Notch signaling pathway is involved in and controls the formation of new blood vessels accompanied with the VEGFA pathway (34). VEGFA, as an upstream regulator of Notch (34), combined with its receptor VEGFR, then induced the expression of Notch 1 and its ligand DII4, and the transcription of Notch target genes were initiated to promote angiogenesis.

The expression of VEGFA is regulated by numerous factors and hypoxia is the strongest known regulator. HIF-1 $\alpha$ is a nuclear transcription factor induced by hypoxia and its expression can be found in all mammalian cells during hypoxia. Experimental studies suggested that following cerebral ischemia, the areas where the expression of HIF-1 $\alpha$ is found is considered to be the region in chronic hypoxia around the infarct area $(35,36)$. HIF-1 $\alpha$ is important in mediating signal transfer between hypoxia and angiogenesis. Hypoxic cells induce the expression of VEGFA via activating HIF-1 $\alpha$ (37). Zhang et al (25) revealed that following focal cerebral ischemia, the expression of VEGFA and VEGFR were significantly increased around the infarcted area. The expression of HIF-1 $\alpha$ was also increased around the infarcted area. Therefore, hypoxia-induced HIF-1 $\alpha$-VEGFA-Notch1 angiogenesis is suggested to be an important pathway in angiogenesis regulation.

In the present study, the in vivo and in vitro results revealed that the expression of HIF-1 $\alpha$ at the mRNA and protein levels were rare in normoxia, however, it was increasingly induced under hypoxia. In addition, the expression of VEGFA and Notch1 were upregulated in the hypoxia group at the mRNA and protein levels compared with the normoxia group. These results illustrated that hypoxia-induced upregulation of VEGFA and Notch1 is associated with the increased expression of HIF-1 $\alpha$.

Studies regarding the regulatory effect of miRNAs on angiogenesis are increasing. Certain studies demonstrated that the expression profiles of a series of miRNAs altered following ischemia, and certain miRNAs are important in angiogen- esis $(20,38,39)$. Therefore, identifying miRNA expression profiles targeting angiogenesis and further studying its regulatory mechanisms are of great significance for the treatment of stroke.

To further examine the role of miR-376b-5p in angiogenesis during cerebral ischemia, the miR-376b-5p mimic and miR-376b-5p inhibitor were transfected into hypoxic HUVECs. The proliferation, migration and tube formation of HUVECs were measured for estimating angiogenesis. The results demonstrated that the miR-376b-5p mimic downregulated angiogenesis and the miR-376b-5p inhibitor upregulated angiogenesis. These results verified that miRNA-376b-5p was able to regulate angiogenesis.

A functional link between miRNA expression and HIF- $1 \alpha$ has been identified by certain studies. HIF- $1 \alpha$ is able to be targeted by the miR-17-92 cluster, miR-424 and miR-20b (40-42). A specific group of miRNAs have been reported to be induced in response to hypoxia, at least partially via a HIF-1-dependent mechanism (16).

The present study predicted the targets of miR-376b-5p by target prediction programs and focused on HIF-1 $\alpha$. Next, to examine the underlying mechanisms responsible for our observation, it was suggested that miRNA-376b-5p regulates angiogenesis via the HIF-1 $\alpha$-VEGFA-Notch1 pathway. HIF-1 $\alpha$ was knocked down by the transfection of shRNA. The results demonstrated that following transfection, the expression of HIF-1 $\alpha$ was low. The miRNA-376b-5p mimic or miR-376b-5p inhibitor was not able to affect angiogenesis via HIF-1 $\alpha$, thus the angiogenesis index, including proliferation, migration, tube formation and the expression of angiogenesis-related molecules VEGFA/Notch1 was significantly different from the mimic/inhibitor group. Furthermore, the present study demonstrated that following shRNA transfection, the angiogenesis index and the expression of angiogenesis-related molecules VEGFA/Notch1 were significantly different from the hypoxia group. It was suggested that since HIF-1 $\alpha$ expression was significantly decreased compared with the hypoxia group; the HIF-1 $\alpha$ mediated signal between hypoxia and angiogenesis was repressed.

In conclusion, the present study systematically demonstrated that miR-376b-5p potently inhibited angiogenesis in the rat MCAO model in vivo and miR-376b-5p was able to effectively inhibit the proliferation, migration and tube formation of HUVECs in vitro. To the best of our knowledge, the present study demonstrated for the first time that miR-376b-5p inhibits angiogenesis in HUVECs by targeting the HIF- $1 \alpha$-mediated VEGFA/Notch1 signaling pathway. In view of the important role of miRNA in the regulation of angiogenesis, identifying new miRNAs targeting angiogenesis and further examining its regulatory pathways and mechanisms, making miRNAs a new target for the treatment of ischemic diseases, are of important instructive significance in vascular repair for the clinical treatment of ischemic stroke.

\section{References}

1. Wang WZ: Neurology. 4th edition. People's Medical Publishing House, Beijing, pp130, 2001.

2. Zhu XF, Rao ML, Peng J, et al: Dynamis observed morphologic change of neuron and microcirculation in focal cerebral ischemia and reperfusion of rat. Chin J Clin Rehabil 6: 1904-1905, 2002. 
3. Bang OY, Saver JL, Buck BH, et al; UCLA Collateral Investigators: Impact of collateral flow on tissue fate in acute ischaemic stroke. J Neurol Neurosurg Psychiatry 79: 625-629, 2008.

4. Liebeskind DS, Cotsonis GA, Saver JL, et al; Warfarin-Aspirin Symptomatic Intracranial Disease (WASID) Investigators: Collaterals dramatically alter stroke risk in intracranial atherosclerosis. Ann Neurol 69: 963-974, 2011.

5. Miteff F, Levi CR, Bateman GA, et al: The independent predictive utility of computed tomography angiographic collateral status in acute ischaemic stroke. Brain 132: 2231-2238, 2009.

6. Krupinski J, Kaluza J, Kumar P, et al: Role of angiogenesis in patients with cerebral ischemic stroke. Stroke 25: 1794-1798, 1994

7. Wu F, Yang Z and Li G: Role of specific microRNAs for endothelial function and angiogenesis. Biochem Biophys Res Commun 386: 549-553, 2009.

8. Longa EZ, Weinstein PR, Carlson S, et al: Reversible middle cerebral artery occlusion without craniectomy in rats. Stroke 20 : 84-91, 1989.

9. Fire A, Xu S, Montgomery MK, et al: Potent and specific genetic interference by double-stranded RNA in Caenorhabditis elegans. Nature 391: 806-811, 1998.

10. Caplen NJ, Parrish S, Imani F, et al: Specific inhibition of gene expression by small doublestranded RNAs in invertebrate and vertebrate systems. Proc Natl Acad Sci USA 98: 9742-9747, 2001

11. Grishok A, Pasquinelli AE, Conte D, et al: Genes and mechanisms related to RNA interference regulate expression of the small temporal RNAs that control C. elegans developmental timing. Cell 106: 23-34, 2001.

12. Bartel DP: MicroRNAs: genomics, biogenesis, mechanism, and function. Cell 116: 281-297, 2004.

13. Ambros V: The functions of animal microRNAs. Nature 431: 350-355, 2004.

14. Humphreys DT, Westman BJ, Martin DI, et al: MicroRNAs control translation initiation by inhibiting eukaryotic initiation factor 4E/cap and poly(A) tail function. Proc Natl Acad Sci USA 102: 16961-16966, 2005.

15. Jing Q, Huang S, Guth S, et al: Involvement of microRNA in AU-rich element-mediated mRNA instability. Cell 120: 623-634, 2005.

16. Kulshreshtha R, Ferracin M, Wojcik SE, et al: A microRNA signature of hypoxia. Mol Cell Biol 27: 1859-1867, 2007.

17. Wen QQ, Jia YJ, Wang MC, et al: Expression analysis of microRNA on acute cerebral ischemia in rats. J Chongqing Univ 33: 23-26, 2005

18. Roy S, Khanna S, Hussain SR, et al: MicroRNA expression in response to murine myocardial infarction: $\mathrm{miR}-21$ regulates fibroblast metalloprotease-2 via phosphatase and tensin homologue. Cardiovasc Res 82: 21-29, 2009.

19. Shen J, Yang X, Xie B, et al: MicroRNAs regulate ocular neovascularization. Mol Ther 16: 1208-1216, 2008.

20. Dharap A, Bowen K, Place R, et al: Transient focal ischemia induces extensive temporal changes in rat cerebral microRNAome. J Cereb Blood Flow Metab 29: 675-687, 2009.

21. Velazquez OC, Snyder R, Liu ZJ, et al: Fibroblast-dependent differentiation of human microvaseular endothelial cells into capillary-like 3-dimensional networks. FASEB J 16: 1316-1318, 2002.

22. Beck H and Plate KH: Angiogenesis after cerebral ischemia. Acta Neuropathol 117: 481-496, 2009.
23. Guo X, Liu L, Zhang M, et al: Correlation of CD34+ cells with tissue angiogenesis after traumatic brain injury in a rat model. J Neurotrauma 26: 1337-1344, 2009.

24. Yancopoulos GD: Vascular-specific growth factors and blood vessel formation. Nature 407: 242-248, 2000

25. Zhang ZG, Zhang L, Jiang Q, et al: VEGF enhances angiogenesis and promotes blood-brain barrier leakage in the ischemic brain. J Clin Invest 106: 829-838, 2000.

26. Risau W: Mechanisms of angiogenesis. Nature 386: 671-674, 1997.

27. Abumiya T, Lucero J, Heo JH, et al: Activated microvessels express vascular endothelial growth factor and integrin alpha(v) beta3 during focal cerebral ischemia. J Cereb Blood Flow Metab 19: 1038-1050, 1999.

28. Sondell M, Lundborg G and Kanje M: Vascular endothelial growth factor has neurotrophic activity and stimulates axonal outgrowth, enhancing cell survival and Schwann cell proliferation in the peripheral nervous system. J Neurosci 19: 5731-5740, 1999.

29. Schratzberger P, Schratzberger G, Silver M, et al: Favorable effect of VEGF gene transfer on ischemic peripheral neuropathy. Nat Med 6: 405-413, 2000.

30. Zhang ZJ and He L: Vascular endothelial growth factor and ischemic cerebrovascular disease. Chin J Clin Rehabil 8: 13197, 2004.

31. Greenberg DA and Jin K: From angiogenesis to neuropathology. Nature 438: 954-959, 2005.

32. Limbourg FP, Takeshita K, Radtke F, et al: Essential role of endothelial Notch1 in angiogenesis. Circulation 111: 1826-1832, 2005.

33. Cai-Li Song, Feng-Chun Zhang, Ying-Chun Xu, et al: Effect of breast cancer stromal cells on expression of Wntl, Notchl and $\beta$-catenin and migration of MCF-7 cells. Medical Bulletin of Shanghai Jiaotong University 28: 921-924, 2008.

34. Hainaud $\mathrm{P}$, Contrerès JO, Villemain A, et al: The role of the vascular endothelial growth factor-Delta-like 4 ligand/Notch4-ephrin B2 cascade in tumor vessel remodeling and endothelial cell functions. Cancer Res 66: 8501-8510, 2006.

35. Jin KL, Mao XO, Nagayama T, et al: Induction of vascular endothelial growth factor and hypoxia-inducible factor-1alpHa by global ischemia in rat brain. Neuroscience 99: 577-585, 2000.

36. Sharp FR, Lu A, Tang Y, et al: Multiple molecular penumbras after focal cerebral ischemia. J Cereb Blood Flow Metab 20: 1011-1032, 2000.

37. Carmeliet P: Mechanisms of angiogenesis and arteriogenesis. Nat Med 6: 389-395, 2000

38. Bonauer A, Carmona G, Iwasaki M, et al: MicroRNA-92a controls angiogenesis and functional recovery of ischemic tissues in mice. Science 324: 1710-1713, 2009.

39. Jeyaseelan K, Lim KY, Armugam A: MicroRNA expression in the blood and brain of rats subjected to transient focal ischemia by middle cerebral artery occlusion. Stroke 39: 959-966, 2008.

40. Taguchi A, Yanagisawa K, Tanaka M, et al: Identification of hypoxia-inducible factor-1 alpha as a novel target for miR-17-92 microRNA cluster. Cancer Res 68: 5540-5545, 2008.

41. Ghosh G, Subramanian IV, Adhikari N, et al: Hypoxia-induced microRNA-424 expression in human endothelial cells regulates HIF-alpha isoforms and promotes angiogenesis. J Clin Invest 120: 4141-4154, 2010.

42. Cascio S, D'Andrea A, Ferla R, et al: miR-20b modulates VEGF expression by targeting HIF-1 alpha and STAT3 in MCF-7 breast cancer cells. J Cell Physiol 224: 242-249, 2010. 